\title{
The existence of equilibria for the rank game
}

\author{
Zhi Lin ${ }^{*}$ \\ Dedicated to Professor Shih-sen Chang on the occasion of his 80th birthday
}

${ }^{*}$ Correspondence: linzhi7525@163.com College of Sciences, Chongqing Jiaotong University, Chongqing, 400074, China

\begin{abstract}
In this paper, we introduce the concept of the rank game and propose a mathematical model for it. By a discrete fixed point theorem, we give the existence results of rank equilibria.
\end{abstract}

MSC: $91 \mathrm{~A} 10 ; 91 \mathrm{~A} 40$

Keywords: rank game; rank-integer; rank-remainder; rank

\section{Introduction}

In $[1,2]$, Nash has proved the existence of an equilibrium for the non-cooperative game by using the fixed point theorem, which is called a Nash equilibrium. In a Nash game each player always maximizes his payoff value and does not pay attention to how much the other player's payoff values are. In other words, each player looks after a high absolute number of his payoff value only and does not care about the rank of his payoff value. But the above assumption is often not true in practice when there exist competitive relationships among players. Since the stronger one is always in an advantageous position in a future game, each player often cares more about his rank of payoff value among all players than the payoff value itself. The game in which each player cares for his rank of payoff value among all players more than the payoff value is said to be a rank game. In this paper, we introduce the concept of the rank game and propose its mathematical model. By a discrete fixed point theorem, we give the existence results of rank equilibria. For other results of the rank game, we refer to [3], and for discrete fixed point theorems, we refer to [4-6] and references therein.

(1) Four basic factors of the rank game.

The rank game belongs to the category of the non-cooperative game. The player, strategy set, and payoff function are three basic factors in a non-cooperative game, thus they are also essential factors in a rank game. Moreover, in a rank game, the payoff of each player is assumed to have an initial value before the game, which is called the initial payoff value of each player. Clearly, the stronger (the bigger of the initial payoff value) and the weaker (the smaller of the initial payoff value) have different options in a rank game; in other words, in a rank game, the choice of each player is not only related to the payoff value in the game, but it also is related to the initial payoff value before the game. Thus the initial payoff value of each player is the fourth key factor in a rank game.

(2) Several basic concepts of the rank game.

\section{基 Springer}

○2014 Lin; licensee Springer. This is an Open Access article distributed under the terms of the Creative Commons Attribution License (http://creativecommons.org/licenses/by/2.0), which permits unrestricted use, distribution, and reproduction in any medium, provided the original work is properly cited. 
Let $I=\{1,2, \ldots, n\}$ denote the set of players. For each $i \in I$, let $K_{i}, f_{i}^{0}$ denote the strategy set and initial payoff value of player $i$, respectively, and let the real function $f_{i}: K \mapsto R$ denote the payoff function of player $i$. Denote $K=\prod_{i=1}^{n} K_{i}, K_{\hat{i}}=\prod_{j \neq i, j=1}^{n} K_{j}$. For each $x \in K$, we can write $x=\left(x_{i}, x_{i}\right)$. For each $i \in I, x \in K$, let $Q_{i}=\left\{j \in I: f_{j}^{0}+f_{j}(x)>f_{i}^{0}+f_{i}(x)\right\}, P_{i}=\{j \in$ $\left.I: f_{j}^{0}+f_{j}(x)=f_{i}^{0}+f_{i}(x)\right\}, m_{i}=\left|Q_{i}\right|+1, l_{i}=\left|P_{i}\right|$, where $|\cdot|$ denotes the element number of the set, and let

$$
h_{i}=\frac{l_{i}-1}{l_{i}}, \quad u_{i}=m_{i}+h_{i} \text {. }
$$

Clearly, $m_{i}$ and $l_{i}$ both are integer and $n \geq m_{i}, l_{i}, u_{i} \geq 1>h_{i} \geq 0$. Denote $f^{0}=\left(f_{1}^{0}, f_{2}^{0}, \ldots\right.$, $\left.f_{n}^{0}\right), f(x)=\left(f_{1}(x), f_{2}(x), \ldots, f_{n}(x)\right)$ (briefly, $\left.f=\left(f_{1}, f_{2}, \ldots, f_{n}\right)\right), F(x)=\left(F_{1}(x), F_{2}(x), \ldots, F_{n}(x)\right)=$ $f^{0}+f(x)=\left(f_{1}^{0}+f_{1}(x), f_{2}^{0}+f_{2}(x), \ldots, f_{n}^{0}+f_{n}(x)\right)$ (briefly, $\left.F=\left(F_{1}, F_{2}, \ldots, F_{n}\right)\right), \forall x \in K$. Note that for each $i \in I, x \in K, u_{i}(x)=u_{i}\left(f^{0}, f(x)\right)$.

Definition 1.1 For each $i \in I, x \in K, m_{i}, h_{i}$, and $u_{i}$ are called the rank-integer, the rank-remainder, and the rank of player $i$ at $x \in K$, respectively. For each $x \in K, u(x)=$ $\left(u_{1}(x), u_{2}(x), \ldots, u_{n}(x)\right)$ (briefly, $\left.u=\left(u_{1}, u_{2}, \ldots, u_{n}\right)\right), f(x)$, and $F(x)$ are called the rank vector, the payoff vector, and the accumulated payoff vector of players at $x \in K$, respectively. If for each $j(n \geq j \geq 1)$, there is $i \in I$ such that $u_{i}=j,\left(u_{1}, u_{2}, \ldots, u_{n}\right)$ is called a complete rank vector, otherwise a noncomplete rank vector of players.

It is easy to verify that for each $i, j \in I, x \in K, u_{i}=(<,>) u_{j}$ if and only if $F_{i}(x)=(>,<) F_{j}(x)$.

(3) The rank game's principle.

The rank game's principle: each player always minimizes the number of players ranking before him (those accumulated payoff values are bigger than that of him) and, after this condition is satisfied, each player always minimizes the number of players ranking the same as he (those accumulated payoff values are the same as that of him), and after these two conditions are satisfied, each player always maximizes his payoff value. Equivalently, the rank game's principle can also be expressed as follows: each player always minimizes the number of players ranking before himself, and after this condition is satisfied, each player always maximizes the number of players ranking after himself (those accumulated payoff values are less than that of his), and after these two conditions are satisfied, each player always maximizes his payoff value.

The rank game is a class of non-cooperative games. Thus, these assumptions of the noncooperative game, for example, each player is rational, the information is symmetrical, and so on, are still necessary in the rank game. The main differences between a Nash game and the rank game are: in a Nash game, each player always maximizes his payoff value and does not care about how much the other player's payoff values are, but in a rank game, each player always minimizes his rank, and after his rank reached minimum, the player always maximizes his payoff value.

A Nash game is suitable for the case that competitive relationships do not exist among players, and the rank game is suitable for the case that there exist competitions among players.

(4) The mathematical model of the rank game.

For each $i \in I$, let $K_{i}, f_{i}^{0}, f_{i}$ denote the strategy set and initial payoff value and payoff function of player $i$, respectively. 
The rank game is: find $x^{*}=\left(x_{1}^{*}, x_{2}^{*}, \ldots, x_{n}^{*}\right) \in K$ such that for each $i \in I, y_{i} \in K_{i}$,

$$
u_{i}\left(f^{0}+f\left(x_{i}^{*}, x_{i}^{*}\right)\right)=\min _{y_{i} \in K_{i}} u_{i}\left(f^{0}+f\left(y_{i}, x_{\hat{i}}^{*}\right)\right)
$$

and if $u_{i}\left(f^{0}+f\left(x_{i}^{*}, x_{\hat{i}}^{*}\right)\right)=u_{i}\left(f^{0}+f\left(y_{i}, x_{\hat{i}}^{*}\right)\right)$, then

$$
f_{i}\left(x_{i}^{*}, x_{\hat{i}}^{*}\right) \geq f_{i}\left(y_{i}, x_{\hat{i}}^{*}\right)
$$

$x^{*}$ is called a rank equilibrium point (briefly, rank equilibrium) of the rank game. A rank game is denoted by $\Gamma=\left\{K_{i}, f_{i}^{0}, f_{i}\right\}_{i \in I}$.

For each $i \in I$, if $K_{i}$ is a finite set, then $f_{i}$ may be denoted by matrices, which are called the payoff matrices; in this case, the rank game $\Gamma$ is called a finite rank game, otherwise an infinite rank game.

\section{Preliminaries}

Throughout this paper, unless otherwise specified, assume that for each $i \in I$, the strategy set is $K_{i}=\left\{1,2, \ldots, k_{i}\right\}$ of player $i$, where $k_{i}$ denotes a positive integer. $K$ is equipped with a sign component-wise order, i.e., $I$ is divided into two subsets (possibly empty) $I_{+}$and $I_{-}$, $\lambda_{j}= \pm 1$ are allocated to $j \in I_{+}$and $j \in I_{-}$respectively, and for each $x=\left(x_{i}\right)_{i \in I}, y=\left(y_{i}\right)_{i \in I} \in K$, $x \preceq y$ is defined by $\lambda_{j} x_{j} \leq \lambda_{j} y_{j}$ for each $j \in I$. For each $i \in I, K_{\hat{i}}=\prod_{j \in I, j \neq i} K_{j}$ is also equipped with the sign component-wise order. It is easy to verify that the sign component-wise order $\preceq$ is a partial order. The symbol $x \prec y$ means $x \preceq y$ and $x \neq y$.

Consider the rank game $\Gamma=\left\{K_{i}, f_{i}^{0}, f_{i}\right\}_{i \in I}$. For each $i \in I$, let $\rho_{i}\left(x_{i}\right)=\left\{x_{i} \in K_{i}: u_{i}\left(f^{0}+\right.\right.$ $\left.\left.f\left(x_{i}, x_{\hat{i}}\right)\right)=\min _{y_{i} \in K_{i}} u_{i}\left(f^{0}+f\left(y_{i}, x_{\hat{i}}\right)\right)\right\}$, and the best responses map $S_{i}: K_{\hat{i}} \mapsto 2^{K_{i}}$ of player $i$ is defined by

$$
S_{i}\left(x_{\bar{i}}\right)=\left\{x_{i} \in K_{i}: f\left(x_{i}, x_{\hat{i}}\right)=\max _{y_{i} \in \rho_{i}\left(x_{\hat{i}}\right)} f_{i}\left(y_{i}, x_{\hat{i}}\right)\right\},
$$

where $2^{K_{i}}$ denotes the family of all nonempty subsets of $K_{i}$.

The best responses map $S: K \mapsto 2^{K}$ of the rank game $\Gamma$ is defined by

$$
S(x)=\prod_{i \in I} S_{i}\left(x_{\bar{i}}\right) .
$$

Note that for each $i \in I, x \in K, \rho_{i}\left(x_{i}\right) \neq \emptyset, S_{i}\left(x_{i}\right) \neq \emptyset$, thus $S(x) \neq \emptyset$. Clearly $x^{*} \in K$ is a rank equilibrium point if and only if $x^{*} \in S\left(x^{*}\right)$.

We have the following lemma; see [4] (Theorem 3.1).

Lemma 2.1 Let $(V, \preceq)$ be a partially ordered set and $g: V \mapsto 2^{V}$ a set-valued map. Assume that

(1) there exist $x^{0} \in V$ and $x^{1} \in g\left(x^{0}\right)$ such that $x^{0} \preceq x^{1}$ and $\left\{x \in V: x^{0} \preceq x\right\}$ is finite;

(2) for each $x \in V$ and $y \in g(x)$,

$$
x \prec y \quad \Rightarrow \quad \exists z \in g(y) \text { s.t. } y \preceq z .
$$

Then $g$ has a fixed point $x^{*} \in g\left(x^{*}\right)$. 


\section{The existence of equilibrium points for the rank game}

Definition 3.1 (Monotonicity) A rank game $\Gamma$ is called monotone if, for each $i \in I, x_{i}^{0}, x_{i}^{1} \in$ $K_{\hat{i}}$ with $x_{\hat{i}}^{0} \prec x_{\hat{i}}^{1}$ and for each $x_{i}^{1} \in S_{i}\left(x_{i}^{0}\right)$, there exists $x_{i}^{2} \in S_{i}\left(x_{i}^{1}\right)$ such that $\lambda_{i} x_{i}^{1} \leq \lambda_{i} x_{i}^{2}$.

Theorem 3.1 Any monotone n-person rank game $\Gamma$ has a rank equilibrium point.

Proof The following proof is analogous to that of Lemma 2.1.

Since $K$ is a finite set and it has a minimum element, the condition (1) of Lemma 2.1 is trivially satisfied. In the following we verify that the condition (2) of Lemma 2.1 is also satisfied.

Assume that $x^{0} \in K$ and $x^{1} \in S\left(x^{0}\right)$ satisfy $x^{0} \prec x^{1}$. Let $I_{1}=\left\{i \in I: x_{i}^{0}=x_{i}^{1}\right\}, I_{2}=\{i \in I$ : $\left.x_{\hat{i}}^{0} \prec x_{i}^{1}\right\}$. Then $I_{1} \cap I_{2}=\emptyset, I=I_{1} \cup I_{2}$. Note that $I_{2} \neq \emptyset$, otherwise $x^{0}=x^{1}$, a contradiction. Assume without loss of generality that $I_{1} \neq \emptyset$. For each $i \in I_{1}$, by $x^{0} \prec x^{1}$, we have $\lambda_{i} x_{i}^{0}<\lambda_{i} x_{i}^{1}$. Take $x_{i}^{2}=x_{i}^{1}$, then $x_{i}^{2}=x_{i}^{1} \in S_{i}\left(x_{i}^{0}\right)=S_{i}\left(x_{i}^{1}\right)$. For each $i \in I_{2}$, we have $x_{i}^{0} \prec x_{i}^{1}$ and $x_{i}^{1} \in S_{i}\left(x_{i}^{0}\right)$, thus, by monotonicity, there exists $x_{i}^{2} \in S_{i}\left(x_{i}^{1}\right)$ such that $\lambda_{i} x_{i}^{1} \leq \lambda_{i} x_{i}^{2}$. Hence $x^{2}=\left(x_{i}^{2}\right)_{i \in I} \in$ $S\left(x^{1}\right)$ and $x^{1} \preceq x^{2}$, i.e., the condition (2) of Lemma 2.1 is satisfied. By Lemma 2.1, there exists $x^{*} \in S\left(x^{*}\right)$.

Definition 3.2 For $i \in I$, a sequence $\left\{x_{i}^{j}\right\}_{j=1}^{t} \subset K_{\hat{i}}$, where $t$ is a positive integer, is called a total-ordered sequence if, for each $j_{1}, j_{2} \in\{1,2, \ldots, t\}$ with $j_{1}<j_{2}$, then $x_{i}^{j_{1}} \prec x_{i}^{j_{2}}$.

Definition 3.3 A sequence $\left\{x^{j}\right\}_{j=1}^{t_{1}}$ is called a subsequence of $\left\{y^{j}\right\}_{j=1}^{t_{2}}$ if $\left\{x^{j}\right\}_{j=1}^{t_{1}} \subset\left\{y^{j}\right\}_{j=1}^{t_{2}}$ and $t_{1}<t_{2}$.

Definition 3.4 For $i \in I$, a sequence $\left\{x_{i}^{j}\right\}_{j=1}^{t} \subset K_{\widehat{i}}$ is called a maximal total-ordered sequence (briefly, MTOS) if: (1) it is a total-ordered sequence; (2) there is no total-ordered sequence $\left\{y_{i}^{j}\right\}_{j=1}^{T} \subset K_{\hat{i}}$ such that $\left\{x_{i}^{j}\right\}_{j=1}^{t}$ is a subsequence of $\left\{y_{i}^{j}\right\}_{j=1}^{T}$.

Definition 3.5 For $i \in I$, let $\left\{x_{\hat{i}}^{j}\right\}_{j=1}^{t} \subset K_{\hat{i}}$ is a MTOS. For each $j \in\{1,2, \ldots, t\}$, let $w_{i}^{j}=$ $\max _{y_{j} \in S_{i}\left(x_{i}^{j}\right)}\left\{y_{j}\right\}, v_{i}^{j}=\min _{y_{j} \in S_{i}\left(x_{i}^{j}\right)}\left\{y_{j}\right\} .\left\{w_{i}^{j}\right\}_{j=1}^{t}$ and $\left\{v_{i}^{j}\right\}_{j=1}^{t}$ are called a best response maximal element sequence (briefly, BRMAES) and a best response minimal element sequence (briefly, BRMIES) of $\left\{x_{i}^{j}\right\}_{j=1}^{t}$, respectively.

Note that for each $i \in I$, there exists at least a MTOS in $K_{\hat{i}}$, and each MTOS has unique BRMAES and BRMIES.

Lemma 3.1 The rank game $\Gamma=\left\{K_{i}, f_{i}^{0}, f_{i}\right\}_{i \in I}$ is monotone iff for each $i \in I$, each BRMAES is nondecreasing when $i \in I_{+}$, and each BRMIES is nonincreasing when $i \in I_{-}$.

Proof ' $\Rightarrow$ ' For each $i \in I$, assume that $\left\{x_{\hat{i}}^{j}\right\}_{j=1}^{t} \subset K_{\widehat{i}}$ is a MTOS.

(1) $i \in I_{+}$, and in the case, $\lambda_{i}=1$. Let $\left\{w_{i}^{j}\right\}_{j=1}^{t}$ denote the BRMAES of $\left\{x_{i}^{j}\right\}_{j=1}^{t}$. For each $j \in\{1,2, \ldots, t-1\}, x_{\hat{i}}^{j} \prec x_{\hat{i}}^{j+1}, w_{i}^{j} \in S_{i}\left(x_{i}^{j}\right)$, since $\Gamma$ is monotone, there exists $\bar{w}_{i}^{j+1} \in S_{i}\left(x_{\hat{i}}^{j+1}\right)$ such that $\lambda_{i} w_{i}^{j} \leq \lambda_{i} \bar{w}_{i}^{j+1}$, i.e., $w_{i}^{j} \leq \bar{w}_{i}^{j+1}$. Note that $\bar{w}_{i}^{j+1} \leq w_{i}^{j+1}$, thus $w_{i}^{j} \leq w_{i}^{j+1}$, i.e., the sequence $\left\{w_{i}^{j}\right\}_{j=1}^{t}$ is nondecreasing.

(2) $i \in I_{-}$, and in the case, $\lambda_{i}=-1$. Let $\left\{v_{i}^{j}\right\}_{j=1}^{t}$ denote the BRMIES of $\left\{x_{i}^{j}\right\}_{j=1}^{t}$. For each $j \in\{1,2, \ldots, t-1\}, x_{\hat{i}}^{j} \prec x_{\hat{i}}^{j+1}, v_{i}^{j} \in S_{i}\left(x_{i}^{j}\right)$, since $\Gamma$ is monotone, there exists $\bar{v}_{i}^{j+1} \in S_{i}\left(x_{\hat{i}}^{j+1}\right)$ such that $\lambda_{i} v_{i}^{j} \leq \lambda_{i} \bar{v}_{i}^{j+1}$, i.e., $v_{i}^{j} \geq \bar{v}_{i}^{j+1}$. Note that $\bar{v}_{i}^{j+1} \geq v_{i}^{j+1}$, thus $v_{i}^{j} \geq v_{i}^{j+1}$, i.e., the sequence $\left\{v_{i}^{j}\right\}_{j=1}^{t}$ is nonincreasing. 
' $\Leftarrow$ ' For each $i \in I$, assume that $x_{\bar{i}} \prec y_{\hat{i}}, x_{i} \in S_{i}\left(x_{\bar{i}}\right)$. There exists a MTOS $\left\{x_{\hat{i}}^{j}\right\}_{j=1}^{t} \subset K_{\widehat{i}}$ such that $x_{\hat{i}}, y_{i} \in\left\{x_{\hat{i}}^{j}\right\}_{j=1}^{t}$. Denote $x_{\hat{i}}=x_{\hat{i}}^{t_{1}}, y_{i}=x_{i}^{t_{2}}$. By $x_{\hat{i}} \prec y_{i}$, we have $t_{1}<t_{2}$.

(1) $i \in I_{+}$and we have the case $\lambda_{i}=1$. Let $\left\{w_{i}^{j}\right\}_{j=1}^{t}$ be the BRMAES of $\left\{x_{i}^{j}\right\}_{j=1}^{t}$ and $w_{i}^{t_{1}} \in$ $S_{i}\left(x_{i}^{t_{1}}\right), w_{i}^{t_{2}} \in S_{i}\left(x_{i}^{t_{2}}\right)$. Since $\left\{w_{i}^{j}\right\}_{j=1}^{t}$ is nondecreasing, we have $w_{i}^{t_{1}} \leq w_{i}^{t_{2}}$. Note that $x_{i} \leq w_{i}^{t_{1}}$, thus $x_{i} \leq w_{i}^{t_{2}}$, i.e., $\lambda_{i} x_{i} \leq \lambda_{i} w_{i}^{t_{2}}$, which implies that the rank game $\Gamma$ is monotone.

(2) $i \in I_{-}$and we have the case $\lambda_{i}=-1$. Let $\left\{v_{i}^{j}\right\}_{j=1}^{t}$ be the BRMIES of $\left\{x_{i}^{j} t_{j=1}^{t}\right.$ and $v_{i}^{t_{1}} \in$ $S_{i}\left(x_{i}^{t_{1}}\right), v_{i}^{t_{2}} \in S_{i}\left(x_{i}^{t_{2}}\right)$. Since $\left\{v_{i}^{j}\right\}_{j=1}^{t}$ is nonincreasing, we have $v_{i}^{t_{1}} \geq v_{i}^{t_{2}}$. Note that $x_{i} \geq v_{i}^{t_{1}}$, thus $x_{i} \geq v_{i}^{t_{2}}$, i.e., $\lambda_{i} x_{i} \leq \lambda_{i} v_{i}^{t_{2}}$, which implies that the rank game $\Gamma$ is monotone.

Theorem 3.2 For the rank game $\Gamma$, assume that each BRMAES is nondecreasing when $i \in$ $I_{+}$and each BRMIES is nonincreasing when $i \in I_{-}$, then for $\Gamma$ there exists a rank equilibrium point.

Proof By Lemma 3.1, the rank game $\Gamma$ is monotone. By Theorem 3.1, the result follows.

Example 3.1 Consider the following 2-persons rank game $\Gamma$, where $K_{1}=K_{2}=\{1,2,3\}$, $f_{1}^{0}=24, f_{2}^{0}=0$, and the payoff matrices are

$$
G_{f}=\left(\begin{array}{ccc}
(15,27) & (23,18) & (35,42) \\
(0,52) & (23,51) & (-17,0) \\
(67,93) & (23,31) & (42,66)
\end{array}\right)
$$

Thus the accumulated payoff matrices are

$$
G_{F}=\left(\begin{array}{ccc}
(39,27) & (47,18) & (59,42)^{R} \\
(24,52) & (47,51) & (7,0) \\
(91,93) & (47,31) & (66,66)
\end{array}\right) .
$$

Take $\left(\lambda_{1}, \lambda_{2}\right)=(-1,1)$. For $i=1$, there exists an unique $\operatorname{MTOS}\{1,2,3\}$ in $K_{\widehat{1}}=K_{2}$. It is easy to verify that $\rho_{1}(1)=\{1\}, S_{1}(1)=\{1\}, \rho_{1}(2)=\{1,3\}, S_{1}(2)=\{1,3\}, \rho_{1}(3)=\{1,2\}, S_{1}(3)=\{1\}$. Thus, the BRMIES $\{v\}=\{1,1,1\}$, and it is a nonincreasing sequence.

For $i=2$, there exists an unique $\operatorname{MTOS}\{3,2,1\}$ in $K_{\widehat{2}}=K_{1}$. It is easy to verify that $\rho_{2}(3)=$ $\{1\}, S_{2}(3)=\{1\}, \rho_{2}(2)=\{1,2\}, S_{2}(2)=\{1\}, \rho_{2}(1)=\{1,2,3\}, S_{2}(1)=\{3\}$. Thus, the BRMAES $\{w\}=\{1,1,3\}$, and it is a nondecreasing sequence.

By Theorem 3.2, for $\Gamma$ there exists a rank equilibrium. It is easy to verify that $(1,3)$ is a rank equilibrium.

Example 3.2 Consider the following 3-persons rank game $\Gamma$, where $K_{1}=K_{2}=K_{3}=\{1,2\}$, $f_{1}^{0}=2, f_{2}^{0}=0, f_{3}^{0}=1$, and the payoff matrices are

$$
\begin{aligned}
& \text { when player } 3 \text { chooses strategy 1, } \quad G_{f}=\left(\begin{array}{cc}
(2,5,1) & (-5,3,-3) \\
(1,2,-2) & (7,8,4)
\end{array}\right), \\
& \text { when player } 3 \text { chooses strategy 2, } \\
& G_{f}=\left(\begin{array}{ll}
(1,4,0) & (0,4,4) \\
(2,2,2) & (0,3,3)
\end{array}\right) .
\end{aligned}
$$


Thus the accumulated payoff matrices are

$$
\begin{aligned}
& \text { when player } 3 \text { chooses strategy } 1, \quad G_{F}=\left(\begin{array}{cc}
(4,5,2) & (-3,3,-2) \\
(3,2,-1) & (9,8,5)
\end{array}\right), \\
& \text { when player } 3 \text { chooses strategy } 2, \quad G_{F}=\left(\begin{array}{cc}
(3,4,1) & (2,4,5) \\
(4,2,3) & (2,3,4)^{R}
\end{array}\right) .
\end{aligned}
$$

Take $\left(\lambda_{1}, \lambda_{2}\right)=(1,1,1)$. For $i=1$, there exist two $\operatorname{MTOS}\{(1,1),(2,1),(2,2)\}$ and $\{(1,1),(1,2)$, $(2,2)\}$ in $K_{\hat{1}}$. It is easy to verify that $\rho_{1}(1,1)=\{2\}, S_{1}(1,1)=\{2\}, \rho_{1}(2,1)=\{2\}, S_{1}(2,1)=\{2\}$, $\rho_{1}(1,2)=\{2\}, S_{1}(1,2)=\{2\}, \rho_{1}(2,2)=\{1,2\}, S_{1}(2,2)=\{1,2\}$. Thus, their BRMAES $\{w 1\}=$ $\{w 2\}=\{2,2,2\}$, and it is a nondecreasing sequence.

For $i=2$, there exist two MTOS $\{(1,1),(2,1),(2,2)\}$ and $\{(1,1),(1,2),(2,2)\}$ in $K_{\widehat{2}}$. It is easy to verify that $\rho_{2}(1,1)=\{1,2\}, S_{2}(1,1)=\{1\}, \rho_{2}(2,1)=\{1,2\}, S_{2}(2,1)=\{2\}, \rho_{2}(1,2)=\{1\}$, $S_{2}(1,2)=\{1\}, \rho_{2}(2,2)=\{2\}, S_{2}(2,2)=\{2\}$. Thus, for MTOS $\{(1,1),(2,1),(2,2)\}$, the BRMAES $\{w 1\}=\{1,2,2\}$, and for MTOS $\{(1,1),(1,2),(2,2)\}$, the BRMAES $\{w 2\}=\{1,1,2\}$. Clearly, $\{w 1\}$ and $\{w 2\}$ are nondecreasing sequences.

For $i=3$, there exist two $\operatorname{MTOS}\{(1,1),(2,1),(2,2)\}$ and $\{(1,1),(1,2),(2,2)\}$ in $K_{\widehat{3}}$. It is easy to verify that $\rho_{3}(1,1)=\{1,2\}, S_{3}(1,1)=\{1\}, \rho_{3}(2,1)=\{2\}, S_{3}(2,1)=\{2\}, \rho_{3}(1,2)=\{2\}$, $S_{3}(1,2)=\{2\}, \rho_{3}(2,2)=\{2\}, S_{3}(2,2)=\{2\}$. Thus, their BRMAES $\{w 1\}=\{w 2\}=\{1,2,2\}$, and it is a nondecreasing sequence.

By Theorem 3.2, for $\Gamma$ there exists a rank equilibrium. It is easy to verify that $(2,2,2)$ is a rank equilibrium.

\section{Competing interests}

The author declares that they have no competing interests.

\section{Acknowledgements}

This work was supported by the National Natural Science Foundation of China (No. 11271389).

Received: 17 February 2014 Accepted: 8 October 2014 Published: 16 Oct 2014

\section{References}

1. Nash, J: Equilibrium points in N-person games. Proc. Natl. Acad. Sci. USA 36, 48-49 (1950)

2. Nash, J: Noncooperative games. Ann. Math. 54, 286-295 (1951)

3. Lin, Z: Rank game. Oper. Res. Fuzziol. 2, 42-52 (2012)

4. Asto, J-i, Kawasaki, H: Discrete fixed point theorems and their application to Nash equilibrium. Taiwan. J. Math. 13(2), 431-440 (2009)

5. Yang, ZF: Discrete fixed point analysis and its applications. J. Fixed Point Theory Appl. 6, 351-371 (2009)

6. Chen, X, Deng, XT: A simplicial approach for discrete fixed point theorems. Algorithmica 53(2), 250-262 (2009) 